\title{
Construction of CuO-modified zeolitic imidazolate framework-9 for photocatalytic hydrogen evolution
}

\author{
Kai Fan a, Zhiliang Jin a,*, Hong Yuan a, Hongyan Hu b,\#, Yingpu Bi b \\ a School of Chemistry and Chemical Engineering, North Minzu University, Yinchuan 750021, Ningxia, China \\ b State Key Laboratory for Oxo Synthesis and Selective Oxidation, Lanzhou Institute of Chemical Physics, Chinese Academy of Sciences, Lanzhou 730000, \\ Gansu, China
}

\section{A R T I C L E I N F O}

\section{Article history:}

Received 1 October 2017

Accepted 31 October 2017

Published 5 December 2017

\section{Keywords:}

ZIF-9

$\mathrm{CuO}$

Eosin $Y$

Sensitized pathway

Hydrogen evolution

Reaction optimization

\begin{abstract}
A B S T R A C T
An efficient CuO-modified zeolitic imidazolate framework-9 (ZIF-9) photocatalyst is successfully prepared at room temperature under mild conditions. It is observed that the ZIF-9/CuO photocatalyst is effective for $\mathrm{H}_{2}$ generation under visible light with sacrificial agent conditions. When the $\mathrm{CuO}$ is introduced, the photocatalytic properties of ZIF-9 are greatly improved and when the content of $\mathrm{CuO}$ is $40 \%$, the photocatalytic activity reaches a maximum of $78.74 \mu \mathrm{mol}$ after $5 \mathrm{~h}$. This results from the 200-300 nm cube structure of ZIF-9 being able to adsorb more dye molecules and the $\mathrm{CuO}$, which connects with ZIF-9, greatly improving the electronic transmission efficiency. Moreover, the interaction between the dye molecule Eosin Y (EY) and the catalyst is also studied by transient fluorescence spectroscopy. A series of characterizations, such as SEM, TEM, XPS, XRD, UV-vis, FTIR, transient fluorescence and photocurrent, are conducted, and the results are in good agreement with the experimental result. In addition, the possible reaction mechanism over EY-sensitized ZIF-9/CuO under visible light irradiation is proposed.
\end{abstract}

(c) 2017, Dalian Institute of Chemical Physics, Chinese Academy of Sciences. Published by Elsevier B.V. All rights reserved.

\section{Introduction}

With the rapid growth of society and the economy, the limited fossil fuel reserves are being depleted at a faster rate. This energy crisis is coupled with a series of environmental pollution problems that arise from fossil energy consumption, which also need to be taken into serious consideration. Therefore, solving the energy and environmental problems have become some of the most pressing tasks at present. Hydrogen, which has the potential as an efficient and safe power resource, has attracted the attention of many researchers in recent years [1-3]. In 1972, a novel type of $\mathrm{TiO}_{2}$ material was found to be a photocatalyst for splitting water to produce hydrogen by Fujishima et al. [4]. It was then found that many different types of semiconductor photocatalysts have the ability to split water to produce hydrogen under visible light conditions, such as the sulfide photocatalysts $\mathrm{MoS}_{2}$ and $\mathrm{WS}_{2}$, g- $\mathrm{C}_{3} \mathrm{~N}_{4}$ and other nitrogen-doped Co-Pi catalysts [5-8]. However, owing to the low use of solar energy, high consumption, low efficiency and other problems, the discovery of a new material to produce hydrogen is of primary importance.

In recent years, a new type of material, metal-organic frameworks (MOFs), has gradually attracted more attention among researchers. The MOF has an opening skeleton frame-

\footnotetext{
* Corresponding author. Tel: +86-13893316102; Fax: +86-951-2067915; E-mail: zl-jin@nun.edu.cn

* Corresponding author. E-mail: HuHongyan@licp.cas.cn

This work was supported by the National Natural Science Foundation of China $(21433007,21603247,41663012)$ and the Ningxia Low-Grade Resource High Value Utilization and Environmental Chemical Integration Technology Innovation Team Project, North Minzu University. DOI: 10.1016/S1872-2067(17)62969-3 | http://www.sciencedirect.com/science/journal/18722067 | Chin. J. Catal., Vol. 38, No. 12, December 2017
} 
work structure (up to $90 \%$ free volume), high specific surface area (beyond $6000 \mathrm{~m}^{2} / \mathrm{g}$ ) and excellent adjustable performance, so it can be applied to many fields, such as gas adsorption, molecular separation, drug delivery and catalysis [9-12]. To be specific, MOFs are a class of crystalline micro-mesoporous hybrid materials that are composed of clusters of a few metallic atoms held in a three-dimensional structure by organic linkers [13]. The diversity and aesthetic structure of the topology are some of the hallmarks of MOF materials. In 2005, more than 12000 MOF structures were reported on the Cambridge Structural Database, and the number of 3D MOFs has been doubling every 3.9 years [14]. Most of the structure is derived from natural minerals and parts are artificially designed. With the continued study of MOF materials, it has been found that MOFs have a certain ability to capture solar energy, and they show great potential as photocatalysts that can be used in water oxidation [15], photocatalytic carbon dioxide reduction [16], visible-light-driven photocatalytic organic reactions [13] and photocatalytic hydrogen production [17]. Compared with the common photocatalysts, the MOF material, with a photocatalytic activity of visible light, has great advantages in terms of porosity and specific surface area, which are beneficial for improving the photocatalytic activity. Under visible light conditions, the basic process of the photocatalytic reaction of the MOF material is that the organic link will act as a photon antenna to generate electrons and holes. In addition, by adjusting the functional groups and the metal center, the chemical and physical properties of the photocatalyst can be easily changed at the molecular level [18]. Zeolitic imidazolate frameworks (ZIFs) are a subclass of MOFs. ZIFs are crystalline three-dimensional networks, which consist of metal ions bridged by metal ions or imidazole-type linkers, and possess attractive structural properties [19]. ZIFs have a higher chemical stability and structural diversity when compared with other MOFs. ZIFs possess several additional attractive features, such as a high surface area arising from their low density and an increased structural customization owing to the presence of organic connectors [20]. Hermes et al. [21] first reported metal@MOF by releasing the metal atoms from the precursors using metalorganic chemical vapor deposition to infiltrate metal atoms into MOFs. Yan et al. [22] reported $\mathrm{AuPd}, \mathrm{MnO}_{x}$, ZIF-8, and graphene composite catalysts for hydrogen production. Furthermore, Koo et al. [23] proposed heterogeneous sensitization of an ultra-small noble metal catalyst-loaded metal oxide (M@MO) applied to semiconductor metal oxide (SMO) nanofibers (NFs) during electrospinning. MOF template catalytic NPs have also been functionalized on SMO NFs through electrospinning followed by calcination. Because of their ultrahigh surface areas, homogeneous active sites and tunable function, MOFs have become an interesting platform for the development of solar energy conversion devices [24].

Especially, the zeolite imidazole framework-9 (ZIF-9), with a hexagonal symmetry calcite topology, is composed of a corner-sharing tetrahedral $\mathrm{CoN}_{4}$ unit, in which the coordination bond between the $\mathrm{Co}^{2+}$ cation and the benzimidazole acid anion is the $\mathrm{N}$ donor ligand [25]. This structure results in ZIF-9 exhibiting good thermal stability and high carbon and nitrogen con- tent. ZIFs are porous structures with a mixed frame composed of metal ions or metal clusters coordinated with organic imidazole or imidazolate ligands. Thus, ZIFs possess many attractive properties, such as high specific surface area and chemically flexible framework [26]. Therefore, it is possible to select a cobalt-based MOF precursor, ZIF-9, as a photocatalyst to split water for the production of hydrogen as described above.

Copper and copper oxide have been studied extensively. Cu-incorporated $\mathrm{TiO}_{2}$ has been regarded as a low-cost photocatalyst with excellent photocatalytic performance for water splitting [27]. Yu et al. [28] studied the $\mathrm{Cu} / \mathrm{ZnS}$ catalyst, which could enhance photocatalytic performance according to firstprinciples calculations. Yu et al. [29] observed that $\mathrm{Cu}_{2} \mathrm{O}$-modified $\mathrm{TiO}_{2}$ could improve the photocatalytic reduction ability. Chang et al. [30] used the piezoresistive effect of $\mathrm{Cu}_{2} \mathrm{O} / \mathrm{CuO}$ nanorods to achieve synergistic photocatalysis. Moreover, a new type of $\mathrm{TiO}_{2}-\mathrm{Ag}$ - $\mathrm{Cu}_{2} \mathrm{O}$ photocatalyst with Z-type structure was successfully synthesized, which exhibited excellent photocatalytic $\mathrm{H}_{2}$ evolution activity [31]. $\mathrm{CuO}$ has been used to improve the photocatalytic efficiency of some wide-bandgap semiconductors owing to it being an important p-type narrow bandgap $\left(E_{\mathrm{g}} \approx 1.2-1.9 \mathrm{eV}\right.$ ) semiconductor $[32,33]$. In the relevant literature, the different composite materials containing $\mathrm{CuO}$ that have been synthesized, such as $\mathrm{TiO}_{2}(\mathrm{Eu}) / \mathrm{CuO}$, $\mathrm{CuO} / \mathrm{TiO}_{2}, \mathrm{CuO}_{x} / \mathrm{TiO}_{2}$ and $\mathrm{Cd}$-doped $\mathrm{CuO}$, have been shown to significantly improve the photocatalytic activity [33-37]. A simpler and more efficient preparation of the $\mathrm{TiO}_{2} / \mathrm{CuO}$ method was reported by Darren et al. [38]. The photocatalyst was synthesized by the impregnation method using P25 (Degussa) as a support, and exhibited a high photocatalytic hydrogen generation activity from a methanol/water solution. Additionally, a highly efficient and stable $\mathrm{Cu}_{2}(\mathrm{OH})_{2} \mathrm{CO}_{3} / \mathrm{TiO}_{2}$ photocatalyst for hydrogen generation was prepared by incorporating $\mathrm{Cu}_{2}(\mathrm{OH})_{2} \mathrm{CO}_{3}$ clusters onto the surface of $\mathrm{TiO}_{2}$ through a facile precipitation method. This photocatalyst exhibited an outstanding photocatalytic activity where the $\mathrm{H}_{2}$-production rate reached $6713 \mu \mathrm{mol} \mathrm{h}^{-1} \mathrm{~g}^{-1}$ [39]. According to the above results, it should be feasible to use $\mathrm{CuO}$ for the synthesis of a new photocatalyst to split water to produce hydrogen.

In this work, a new ZIF-9 material with excellent openframework structure was successfully prepared at room temperature by a mild conditions method. Then, under the condition of light irradiation, the copper oxide was deposited on the ZIF-9 surface in methanol solution. The photocatalyst ZIF-9/CuO was characterized, and the presence of the catalyst was well demonstrated. Furthermore, the effects of different conditions on the photocatalyst activity were studied. A possible mechanism for the photocatalytic hydrogen production is also discussed.

\section{Experimental}

\subsection{Photocatalyst preparation}

The chemicals used in the experiment were of analytical grade and were used without any further purification. The preparation of ZIF-9 was carried out at room temperature. 
First, $\mathrm{Co}\left(\mathrm{NO}_{3}\right)_{2} \cdot 6 \mathrm{H}_{2} \mathrm{O}(1.46 \mathrm{~g})$ was mixed into $70 \mathrm{~mL}$ methanol and then $3.3 \mathrm{~g}$ 2-methylimidazole was also mixed into methanol $(70 \mathrm{~mL})$. Stirring was continued until the solids in the solutions were completely dissolved. Second, the completely dissolved solution of 2-methylimidazole was slowly added to $\mathrm{Co}\left(\mathrm{NO}_{3}\right)_{2} \cdot 6 \mathrm{H}_{2} \mathrm{O}$ solution. Finally, the mixture was stirred at room temperature for $24 \mathrm{~h}$ and then centrifuged. The resulting solid was washed three times with methanol $(50 \mathrm{~mL})$, and dried overnight at $75^{\circ} \mathrm{C}[40]$.

The prepared ZIF-9 ( $0.2 \mathrm{~g}$ ) was dissolved in a 50\% methanol solution, and a certain mass fraction of $\mathrm{CuCl}_{2}$ was added and stirring was continued for a certain period of time under irradiation conditions. The solution was filtered, washed three times with methanol solution and then dried overnight at $80^{\circ} \mathrm{C}$.

In the typical synthesis of the ZIF-9/CuO photocatalyst, the ZIF-9/CuO photocatalyst with different CuO contents $(20 \%$, $30 \%, 40 \%, 50 \%$, and $60 \%$, respectively) was synthesized by the same method. For comparison, $\mathrm{CuCl}_{2}$ was not added during the preparation of a set of catalysts and the remaining conditions were the same, which was referred to as the blank group.

Similarly, the effect of different irradiation times on the ZIF-9/CuO $40 \%$ photocatalyst was also studied, and the illumination times were $1.5,2.5,3.5,4.5$, and 5.5 h. For comparison, the pure ZIF-9 was also studied.

\subsection{Characterization}

The morphologies and microstructures of the as-prepared samples were characterized by using field-emission scanning electron microscopy (SEM) at an accelerating voltage of $5.0 \mathrm{kV}$. The sample was added dropwise to the copper holder, and the gold plating was carried out to improve the conductivity of the sample. High-resolution transmission electron microscopy (HRTEM) characterization was performed using a FEI Tecnai TF20 high-resolution transmission electron microscope with an accelerating voltage of $300 \mathrm{kV}$. X-ray photoelectron spectroscopy (XPS, ESCALAB 250Xi) measurements were performed to detect the elemental composition. The fluorescence spectra were obtained using a FluoroMAX-4 spectrometer (HORIBA Scientific, France) at room temperature. X-ray diffraction (XRD) crystalline structure analysis was performed using $\mathrm{Cu} K_{\alpha}$ radiation (tube current $40 \mathrm{~mA}$, tube voltage $40 \mathrm{kV}$ ). The Fourier transform infrared (FT-IR) spectra were recorded using a Perkin-Elmer Spectrum 100 IR Fourier transform infrared spectrometer. The UV-visible diffuse reflectance (bandgap) was measured using a UV-2550 (Shimadzu) spectrometer. The photoelectrochemical experiments were carried out on a Princeton electrochemical workstation (PAR VersaSTAT 4) using a standard three-electrode system for analysis. The saturated calomel electrode (SCE) was employed as the reference electrode, a Pt electrode was employed as counter electrode, and the electrolyte was a $\mathrm{Na}_{2} \mathrm{SO}_{4}$ aqueous solution $(0.1 \mathrm{~mol}$ $\mathrm{L}^{-1}$ ). The soaking area of the working electrode in the electrolyte was $1 \mathrm{~cm}^{2}$, and the light source was a $300 \mathrm{~W}$ xenon lamp with a $420 \mathrm{~nm}$ cut-off filter to filter out ultraviolet light.

\subsection{Photocatalytic $\mathrm{H}_{2}$ evolution experiments}

The photocatalytic experiment was carried out by a Prefectlight PCX50A multipass light catalytic reaction system. In this representative photocatalytic experiment, the catalyst $(10 \mathrm{mg})$ was added to a $15 \%(v / v)$ triethanolamine $(30 \mathrm{~mL}, \mathrm{TEOA})$ aqueous solution. Eosin Y (20 mg) dye was then added and stirred for approximately $30 \mathrm{~min}$ by a magnetic stirrer to allow the EY to be completely adsorbed on the catalyst. The reaction bottle had a cap with a rubber stopper, which formed a closed space. The reaction mixture was ventilated with high purity $\mathrm{N}_{2}$, and then, followed by continuous irradiation under magnetic stirring conditions, the photocatalytic hydrogen production activity was measured. The amount of hydrogen evolution was measured using gas chromatography (Tianmei GC7900, TCD, 13X packed column, $\mathrm{N}_{2}$ as carrier).

Before the start of the experiment, all glassware was strictly cleaned and washed with ultrapure water.

\section{Results and discussion}

\subsection{Morphology and structure}

Fig. 1 showed the morphologies of pure ZIF-9, blank group, pure $\mathrm{CuO}$ and $\mathrm{ZIF}-9 / \mathrm{CuO} 40 \%$ samples. It can be seen that the crystal structure of pure ZIF-9 was mainly a hexagonal block-like cube structure. However, the structure of the blank group after the methanol solution and the light irradiation treatment was similar to that of a sphere, and its surface was uneven. Fig. 1(C) shows the image of pure $\mathrm{CuO}$, which consisted of elongated irregular flakes. Moreover, when $\mathrm{CuO}$ was added to ZIF-9, it was observed that the flaky $\mathrm{CuO}$ and the globular ZIF-9 overlapped with each other.

To further understand the microstructure of the photocatalyst, TEM was also employed (Fig. 2). It revealed that the pure ZIF-9 possessed a cube structure, which was approximately 200-300 nm. The image of the ZIF-9/CuO 40\% clearly revealed that the copper oxide and ZIF-9 were interconnected together. The structure of ZIF-9 was obviously changed. The elements

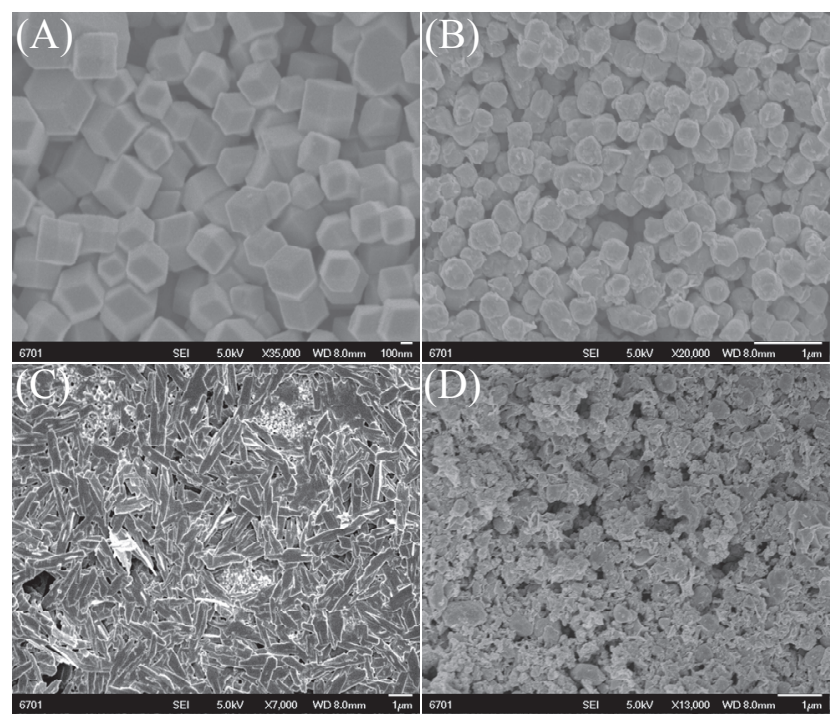

Fig. 1. SEM images of the as-obtained samples. (A) Pure ZIF-9; (B) Blank group; (C) CuO; (D) ZIF-9/CuO 40\%. 

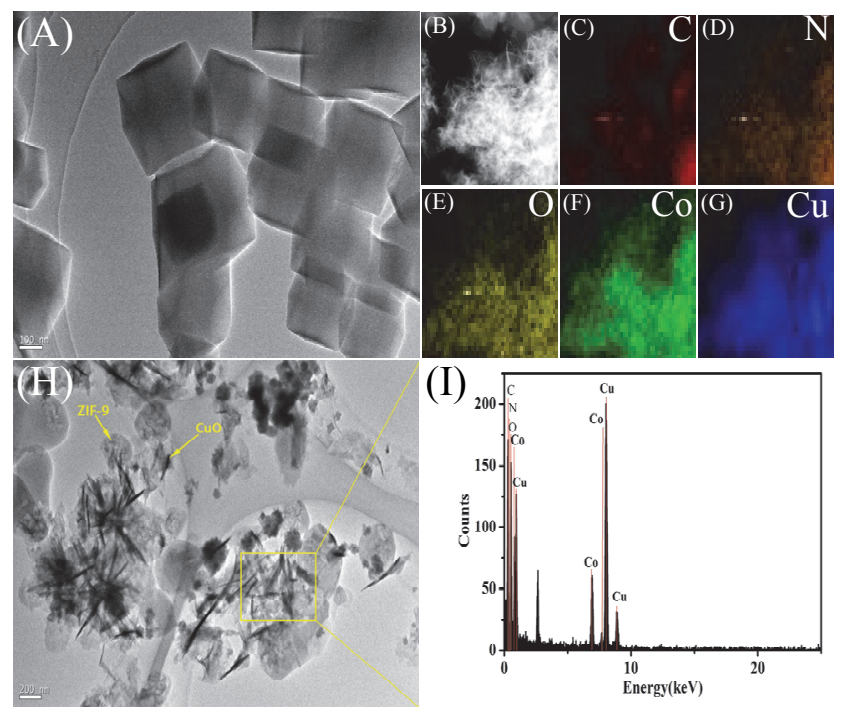

Fig. 2. TEM images of pure ZIF-9 (A), ZIF-9/CuO 40\% (B, H), EDS elemental mapping images $(\mathrm{C}-\mathrm{G})$ taken from $(\mathrm{B})$, and the EDX images (I) taken from a selected area in $(\mathrm{H})$.

that made up the catalyst were identified by EDS elemental mapping, which was taken from the area shown in Fig. 2(B). Energy dispersive X-ray spectroscopy (EDX) was also performed. From the results, the $\mathrm{C}, \mathrm{N}, \mathrm{O}, \mathrm{Co}$ and $\mathrm{Cu}$ elements were present in the yellow region of the ZIF-9/CuO 40\% (Fig. 2(F)). Furthermore, this result was consistent with the XPS characterization results.

To study the crystal structure of the ZIF-9/CuO photocatalyst and determine whether the catalyst was successfully synthesized, the samples were characterized by XRD. The patterns of pure ZIF-9, ZIF-9/CuO 20\%, ZIF-9/CuO 40\% and ZIF-9/CuO $60 \%$ are shown in Fig. 3(A). The pure ZIF-9 patterns exhibited three typical peaks. The strongest diffraction peak at $7.32^{\circ}$ could be assigned to the (100) plane, and the interplanar spacing was $12.07 \mathrm{~nm}$. The other main peaks at $10.36^{\circ}$ and $12.70^{\circ}$ corresponded to the (110) and (111) diffraction signals. A similar XRD pattern has been observed from a previous report [41]. By comparison, it was shown that after treatment and adding $\mathrm{CuO}$, the phase of the catalyst did not change. However, perhaps as a result of the low doped percentages of copper and the small particles dispersed on the catalyst, the diffraction peaks of copper oxide were not observed. The XRD patterns of the photocatalyst ZIF-9/CuO 40\% before and after the reaction are shown in Fig. 3(B). It was found that the XRD pattern did not change before and after the reaction, which indicated that the crystal form did not change.

To further understand the information of the catalyst surface and the element valence characteristics of the photocatalyst, XPS analysis was employed. The results are shown in Fig. 4. The survey spectrum of the ZIF-9/CuO $40 \%$ sample mainly contained $\mathrm{C}, \mathrm{N}, \mathrm{O}$, Co and $\mathrm{Cu}$ elements, and this result was in agreement with the EDX results (Fig. 2(I)). In the $\mathrm{C} 1 s$ spectrum with two characteristic peaks, the position of the characteristic peak at $284.8 \mathrm{eV}$ could be assigned to carbon $s p^{2}$ in the phenyl of the catalyst or amorphous carbon. The peak at $288.6 \mathrm{eV}$ could be attributed to the $\mathrm{C}-\mathrm{N}-\mathrm{H}$ and $\mathrm{C}-\mathrm{N}-\mathrm{C}$ functional groups in the ZIF-9/CuO structure (Fig. 4(B)). The N 1s spectrum exhibited one peak located at $399.14 \mathrm{eV}$, which represented the tertiary $\mathrm{N}$ bonded to carbon atoms in the form of $\mathrm{N}-(\mathrm{C})_{3}$ or $\mathrm{H}-\mathrm{N}-(\mathrm{C})_{2}$. In Fig. 4(D), the $\mathrm{O} 1 \mathrm{~s}$ spectrum presented a single peak at $531.87 \mathrm{eV}$, which corresponded to the surface adsorbed hydroxyl. As shown in Fig. 4(E), the Co $2 p$ spectrum could be deconvoluted into two peaks for Co $2 p_{1 / 2}$ and Co $2 p_{3 / 2}$ located at around 794.68 and $779.3 \mathrm{eV}$, which suggested that the Co existed in the form of a $\mathrm{Co}^{2+}$ valence state. The binding energies of 954.67 and $934.94 \mathrm{eV}$ belonged to $\mathrm{Cu} 2 p_{1 / 2}$ and $\mathrm{Cu} 2 p_{3 / 2}$ (Fig. $4(F))[42,43]$. From the above results, it was determined that $\mathrm{Cu}$ was present in the divalent form.

It is well known that a high surface area and macroporous structure are favorable for improving the photocatalytic activity, because they can result in more adsorption of reactant molecules, and are also more conducive to the transport of reactants and electron transfer. Fig. 5 and Table 1 show the results of the as-prepared pure ZIF-9, blank group and ZIF-9/CuO 40\% showing the specific surface area and pore structure by the nitrogen adsorption isotherms. The surface area for the pure ZIF-9, blank group and ZIF-9/CuO 40\% samples were 1423.94, 1299.07 and $110.52 \mathrm{~m}^{2} \mathrm{~g}^{-1}$, respectively. When $\mathrm{CuO}$ was deposited on the surface of ZIF-9, the specific surface area was reduced but was still greater than $100 \mathrm{~m}^{2} \mathrm{~g}^{-1}$. Moreover, the pore sizes of the samples were 1.9740, 2.1273 and $8.7185 \mathrm{~nm}$, respectively, which showed that they were mesoporous materials. The pore size distribution curve, calculated according to

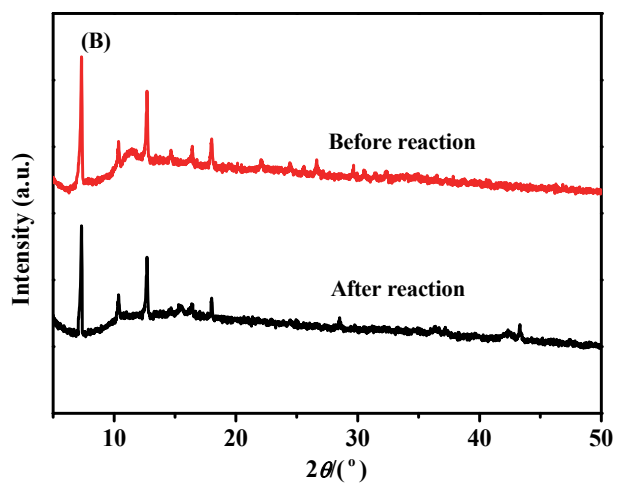

Fig. 3. (A) XRD patterns of pure ZIF-9, ZIF-9/CuO 20\%, ZIF-9/CuO 40\% and ZIF-9/CuO 60\%; (B) XRD patterns of ZIF-9/CuO 40\% before and after reaction. 

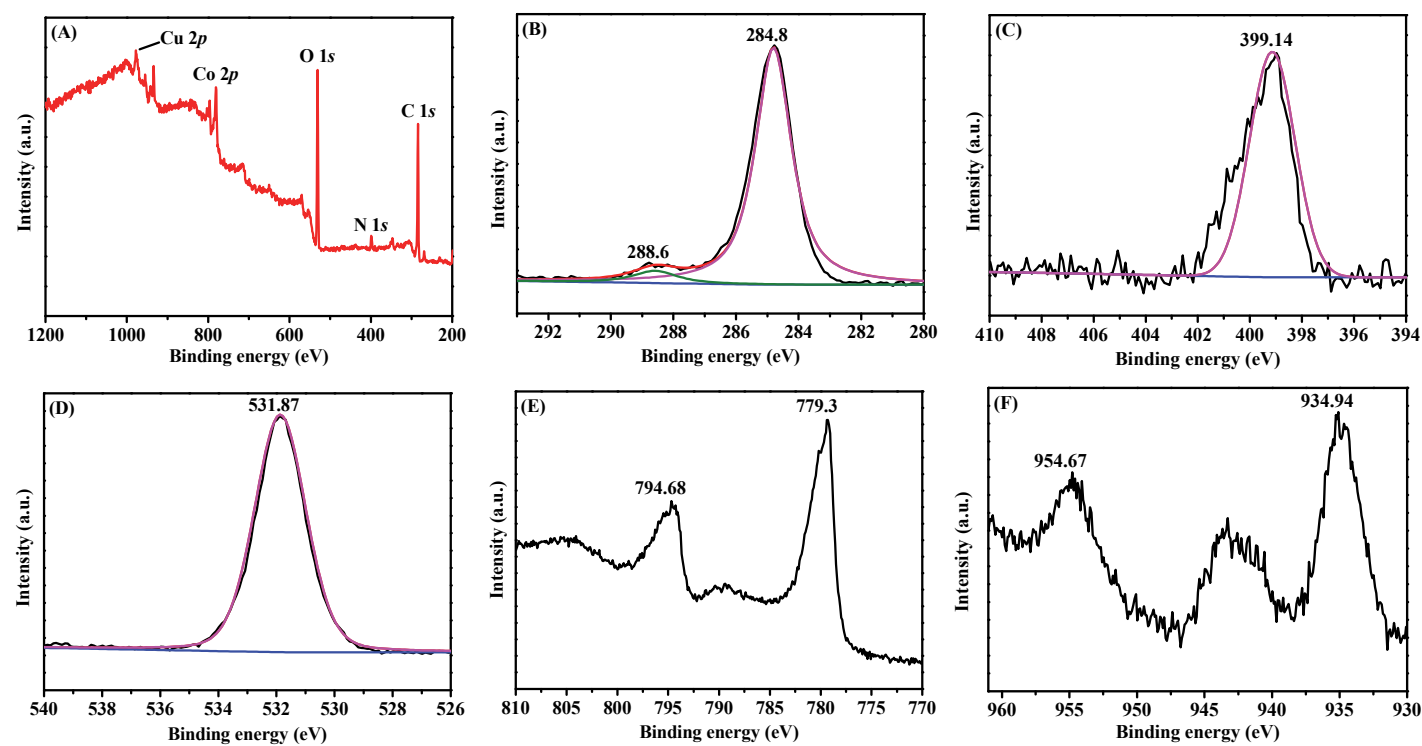

Fig. 4. (A) XPS survey spectra of the ZIF-9/CuO 40\% sample; C $1 s$ (B), N $1 s$ (C), O $1 s$ (D), Co $2 p$ (E) and Cu $2 p$ (F) scan spectra of the ZIF-9/CuO 40\% sample.

the Barrett-Joyner-Halenda (BJH) method, is shown in the inset of Fig. 5. This indicated a wide pore diameter distribution in the range of 0-20 nm. Therefore, the addition of CuO to ZIF-9 having a large specific surface was useful for improving the photocatalytic activity.

\subsection{Photocatalytic performance}

Fig. 6(A) shows the hydrogen production activity curve of an EY-sensitized ZIF-9, ZIF-9/CuO (Cu contents were 20\%, $30 \%, 40 \%, 50 \%, 60 \%)$ system under visible light irradiation $(\lambda$ $\geq 420 \mathrm{~nm}$ ). The hydrogen production of pure ZIF-9 under visible light irradiation for $5 \mathrm{~h}$ could only reach $5.33 \mu \mathrm{mol}$, which indicated that the hydrogen production activity of the pure ZIF-9 catalyst was limited under visible light irradiation. The proposed reason was as a result of the limited efficiency of photogenerated electron hole separation and the low efficiency of electronic transmission, which meant that only a few electrons escaped from the inside to the surface of the catalyst.

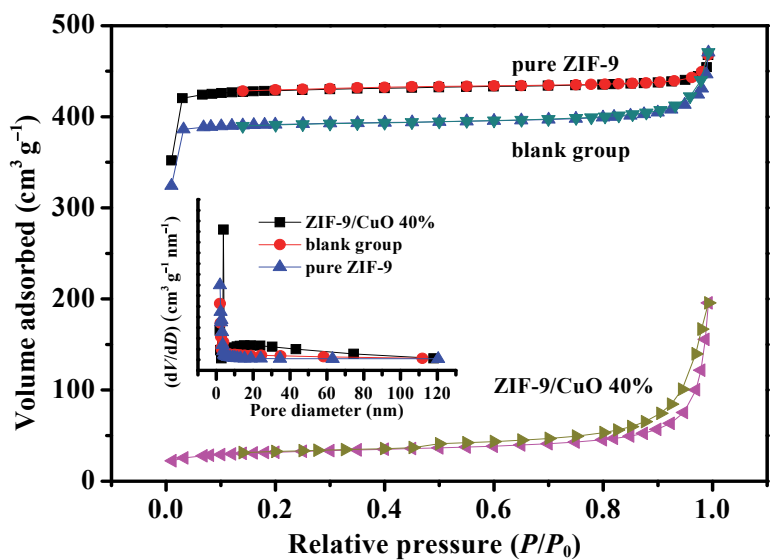

Fig. 5. Nitrogen adsorption-desorption isotherms and corresponding pore size distribution curves (insert) of pure ZIF-9, blank group and ZIF-9/CuO 40\%.
Accordingly, the combined probability that the electrons and $\mathrm{H}^{+}$ existed in the reactive solution was very low, which resulted in minimal hydrogen production. When the pure ZIF-9 was treated with methanol solution and irradiated for the same times, Fig. 6(A) shows that the activity of photocatalytic hydrogen production was improved. The most direct evidence was that the hydrogen production of the catalyst was approximately double that of pure ZIF-9 under the same time of visible light irradiation. The morphological structure of the photocatalyst underwent some changes in the process of deposition (Fig. 6). When adding metal oxide $\mathrm{CuO}$ to ZIF-9 to prepare the ZIF-9/CuO photocatalyst, it could be clearly seen that the photocatalytic activity of the catalyst was dramatically enhanced under the same conditions. When the $\mathrm{CuO}$ content was $20 \%$, $30 \%, 50 \%$ and $60 \%$, the hydrogen production was 48.44 , $67.45,63.45$ and $44.31 \mu \mathrm{mol}$, respectively. When the $\mathrm{CuO}$ content was $40 \%$, the amount of hydrogen production reached the maximum value of $78.74 \mu \mathrm{mol}$. A possible reason for the phenomenon was that Co-ZIF-9 has an open-framework structure that provides a limited number of attachment positions for $\mathrm{CuO}$. Moreover, the amount of $\mathrm{CuO}$ could have a beneficial effect on the system and could promote the improvement of the photocatalytic activity to a certain extent. However, $\mathrm{CuO}$ will accumulate on the ZIF-9 owing to the addition of an excess of copper, which will result in reduced contact between the catalyst and the TEOA solution. Hence, the electrons that escaped from the inside to the surface will first be consumed by the

Table 1

Nitrogen isothermal adsorption characterization.

\begin{tabular}{lccc}
\hline Sample & $\begin{array}{c}S_{\mathrm{BET}^{\mathrm{a}}} \\
\left(\mathrm{m}^{2} \mathrm{~g}^{-1}\right)\end{array}$ & $\begin{array}{c}\text { Pore volume } \\
\left(\mathrm{cm}^{3} \mathrm{~g}^{-1}\right)\end{array}$ & $\begin{array}{c}\text { Average pore } \\
\text { size }^{\mathrm{b}}(\mathrm{nm})\end{array}$ \\
\hline Pure ZIF-9 & 1423.94 & 0.7027 & 1.9740 \\
blank group & 1299.07 & 0.6909 & 2.1273 \\
ZIF-9/CuO 40\% & 110.52 & 0.2408 & 8.7185 \\
\hline
\end{tabular}

a Obtained from BET method. ${ }^{\mathrm{b}}$ Total pore volume taken from the $\mathrm{N}_{2}$ adsorption volume at a relative pressure $\left(P / P_{0}\right)$ of 0.99 . 

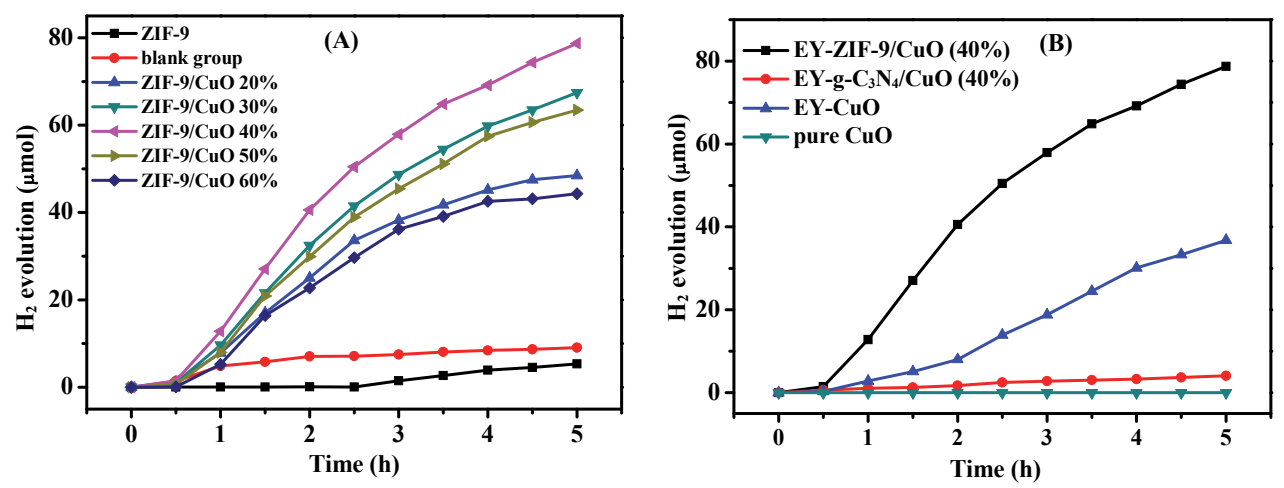

Fig. 6. Under visible light irradiation $(\lambda \geq 420 \mathrm{~nm})$, hydrogen production curves of Eosin (20 mg) sensitized ZIF-9, ZIF-9/CuO (10 mg) (A) and pure $\mathrm{CuO}, \mathrm{EY}-\mathrm{CuO}, \mathrm{g}-\mathrm{C}_{3} \mathrm{~N}_{4} / \mathrm{CuO}$ and $\mathrm{ZIF}-9 / \mathrm{CuO}(\mathrm{B})$ in $15 \%$ TEOA aqueous solution ( $\mathrm{pH}=10$ and reaction time $5 \mathrm{~h}$ ).

excess copper, and only a small number of electrons will combine with the $\mathrm{H}^{+}$in the solution, which will result in a decrease in hydrogen production. Through the study of hydrogen production activity, we observed that a metal oxide $(\mathrm{CuO})$ could effectively improve the hydrogen production activity and we also indirectly confirmed the existence of the ZIF-9/CuO system.

The activity of hydrogen production of different main catalysts is shown in Fig. 6, to investigate the influence of different materials on the photocatalytic system. Under the premise of the addition of the same amount of $\mathrm{CuO}$, we took ZIF-9 and g- $\mathrm{C}_{3} \mathrm{~N}_{4}$ as the main catalysts. In addition, the pure $\mathrm{CuO}$ and EY-CuO were also tested. It was observed that pure $\mathrm{CuO}$ had a very low hydrogen production activity in the absence of EY; however, when EY was added, its activity was improved. The catalytic activity was also changed when $\mathrm{CuO}$ was attached to g- $\mathrm{C}_{3} \mathrm{~N}_{4}$ or ZIF-9. The catalytic activity of ZIF-9/CuO (40\%) was further improved by sensitization of EY, while the activity of g- $\mathrm{C}_{3} \mathrm{~N}_{4} / \mathrm{CuO}(40 \%)$ was inhibited. Under the same treatment, the hydrogen production of $\mathrm{g}_{3} \mathrm{C}_{3} \mathrm{~N}_{4} / \mathrm{CuO}(40 \%)$ only reached $3.62 \mu \mathrm{mol}$, whereas the hydrogen production of ZIF-9/CuO (40\%) was 19.4 times more than that of $\mathrm{g}^{-} \mathrm{C}_{3} \mathrm{~N}_{4} / \mathrm{CuO}(40 \%)$. The only difference was in the test of a different carrier material. The possible reasons for this were the specific surface area and the fact that these two compounds have different morphological structures. Compared with the layered structure of g- ${ }_{3} \mathrm{~N}_{4}$ [44], the ZIF-9 exhibited an open-type sodium-sodium topological structure that could provide more sites for copper oxide (CuO). This could supply a more efficient electro transport channel, which directly impacted on the hydrogen production.

To study the influence of the deposition time on the hydrogen production activity of the catalyst, the deposition time was changed under the same experimental conditions. Fig. 7 shows that after mixing ZIF-9 with $\mathrm{CuCl}_{2}$ with light irradiation deposition for $1.5 \mathrm{~h}$, the hydrogen production of the prepared catalyst reached $60.59 \mu \mathrm{mol}$. When the deposition time was $2.5 \mathrm{~h}$, the hydrogen production activity of the catalyst reached the maximum value. However, with the further increase of the deposition time from 2.5 to $5.5 \mathrm{~h}$, the color of the catalyst gradually deepened; however, the activity of photocatalytic hydrogen production gradually decreased. The possible cause of this phenomenon was that when the deposition time was only $1.5 \mathrm{~h}$, the copper oxide was not sufficiently attached to the surface of ZIF-9. As a result, the activity of hydrogen production was relatively low. When the deposition time was $2.5 \mathrm{~h}$, the metal copper could receive sufficient irradiation so that it could effectively and fully adhere to the surface of ZIF-9. Nevertheless, a further increase of the deposition time resulted in a higher degree of the disruption of ZIF-9 in the methanol solution, and fewer sites were available to connect to $\mathrm{CuO}$.

According to the literature, TEOA, a sacrificial electron donor, plays an important role in the hydrogen production of photocatalysts [45]. Especially, when the $\mathrm{pH}$ value of a TEOA solution is changed, this has a great influence on the efficiency of photocatalytic hydrogen production. The change of $\mathrm{pH}$ value of a TEOA solution can influence the forms of the dye molecule and sacrificial agent molecule and can also influence the redox potential of the semiconductor and the adsorption behavior between the dye molecule and the adsorbed substrate. Moreover, the presence of a sacrificial electron donor is essential for the regeneration of a sensitizer [46,47]. Fig. 8 shows the photocatalytic hydrogen production activity of the reaction system when the $\mathrm{pH}$ of the TEOA solution was changed. A negative influence on the photocatalytic activity for hydrogen production was observed in a weak acid environment. In an alkaline environment, with an increase of the $\mathrm{pH}$ value, the activity of hydrogen production will first increase and then decrease. In comparison, an alkaline environment is beneficial to the catalytic activity of the system. When $\mathrm{pH}=10$, the photocatalytic

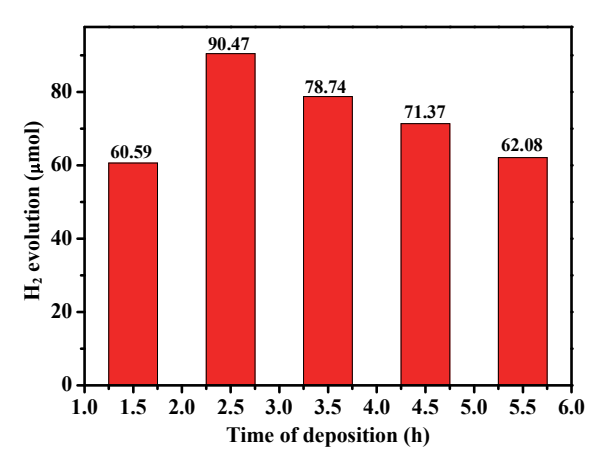

Fig. 7. Influence of deposition time on photocatalytic activity of the ZIF-9/CuO. Reaction conditions: $\mathrm{CuO}$ content $40 \%$, reaction time $5 \mathrm{~h}$. 


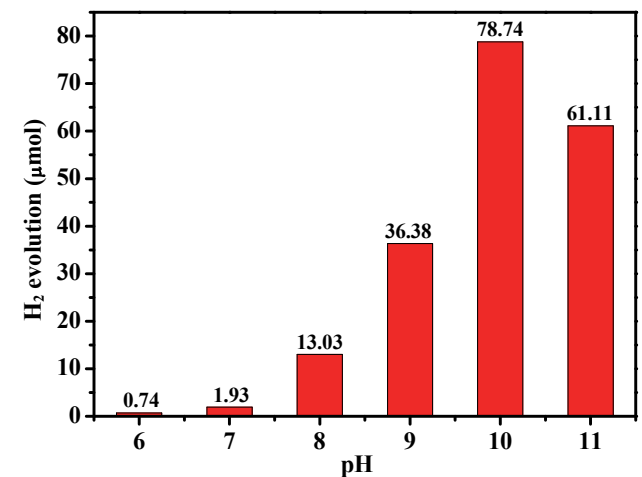

Fig. 8. Influence of different $\mathrm{pH}$ of TEOA solutions on photocatalytic activity of the ZIF-9/CuO. Reaction conditons: $\mathrm{CuO}$ content $40 \%$, reaction time $5 \mathrm{~h}$.

activity was the highest with the hydrogen production amount reaching $78.74 \mu \mathrm{mol}$ after $5 \mathrm{~h}$. The reasons for this phenomenon can be explained as follows: when $\mathrm{pH} \leq 7$, the TEOA solution will be protonated, and the ability to supply electrons is weakened, which leads to the decrease in the photocatalytic activity. In contrast to the acidic environment, TEOA may be in an alkaline environment, and an EY dye molecule can be adsorbed on the catalyst surface in this environment. However, under acidic conditions, the photocatalytic hydrogen production is limited, so the catalytic activity is strong only in an alkaline environment.

Fig. 9 shows the stability of the EY-ZIF-9/CuO system test results. The gas in the reaction system was replaced before the commencement of each cycle. The second and third reaction cycles showed a gradual decrease in the hydrogen production performance of the system. The hydrogen production in the second and third cycles was $81.11 \%$ and $54.12 \%$ of the first cycle, respectively. However, at the beginning of the fourth cycle, the same amount of eosin dye molecules (20 mg) was added. The results show that the hydrogen production amount increased, and the performance was approximately $77.41 \%$ of the first cycle. In addition, the hydrogen production performance of the fifth cycle was also reduced. By comparison, it was observed that the degradation of eosin dyes led to the decline in hydrogen production. Therefore, eosin dyes are key factors that affect the efficiency of hydrogen production [48]. Overall, the catalytic hydrogen production capacity of the system de-

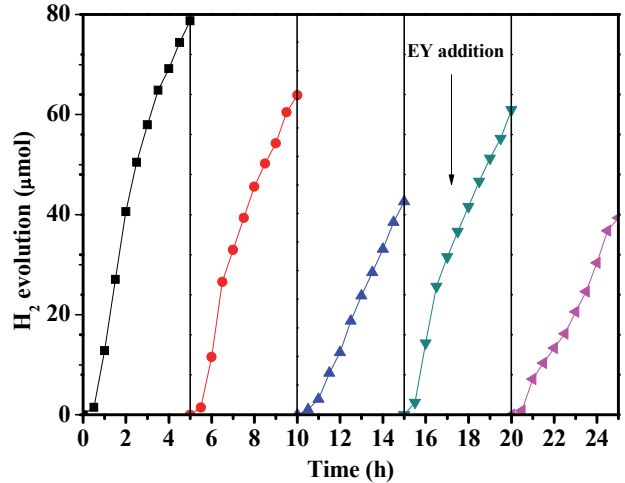

Fig. 9. Stability of photocatalytic $\mathrm{H}_{2}$ evolution over the EY-ZIF-9/CuO system.

creased slightly but could be maintained within a certain range. This arose from the disruption of ZIF-9 in the reaction solution.

Fig. 10 shows the FT-IR spectra of the ZIF-9, blank group and ZIF-9/CuO 40\% samples. According to the literature, there is a great difference between the FT-IR spectra of ZIF-9 and 2-methylimidazole. In the 3300 to $2200 \mathrm{~cm}^{-1}$ range, different intensities of the peak intensities can be observed in the FT-IR spectra, which indicate the presence of an $\mathrm{N}-\mathrm{H} \cdots \mathrm{N}$ hydrogen bond [41]. Meanwhile, many different absorption peaks exist simultaneously in the FT-IR spectrum of ZIF-9: the broad absorption peak at $3400 \mathrm{~cm}^{-1}$ is the stretching vibration peak of $-\mathrm{OH}$ in a water molecule; the absorption peak at $1410 \mathrm{~cm}^{-1}$ should be $-\mathrm{OH}$, which belongs to the $\mathrm{C}-\mathrm{OH}$ group; the $\mathrm{C}-\mathrm{O}$ stretching vibration peak should be at approximately 1200 $\mathrm{cm}^{-1}$; and the absorption peak of $\mathrm{C}-\mathrm{H}$ also appears at 984 and $747 \mathrm{~cm}^{-1}$. By comparing the spectra of the three samples treated by different methods, we observed that except for the change in intensity of each absorption peak, the spectra were almost the same. Therefore, we could conclude that in the process of deposition, the basic structure of ZIF-9 was not destroyed.

The light absorption capacity of the photocatalyst could be determined by UV-vis spectroscopy; moreover, the light absorption capacity of the photocatalyst directly affected the photocatalytic activity. The UV-vis diffuse reflection spectra of pure ZIF-9, blank group and ZIF-9/CuO 40\% complexes are shown in Fig. 10(B). An absorption edge was observed in the near ultraviolet region where the characteristic bands were
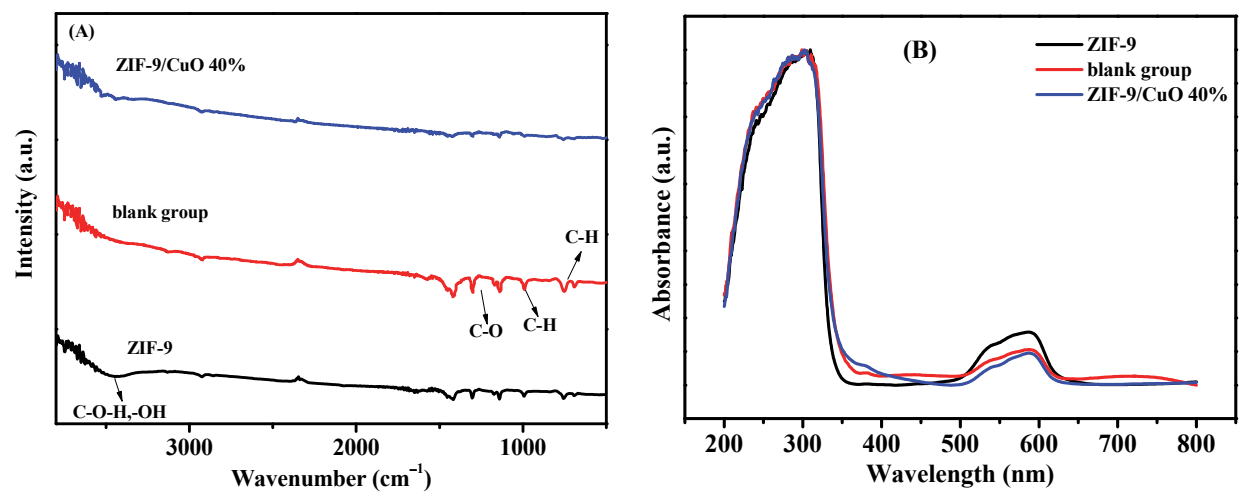

Fig. 10. FT-IR spectra (A) and the UV-vis diffuse reflection spectra (B) of pure ZIF-9, blank group and ZIF-9/CuO 40\% complexes. 
located at 350 to $400 \mathrm{~nm}$, and there was also an absorption edge in the visible range with characteristic bands at 600 to $650 \mathrm{~nm}$. The photon energy absorbed by the transition metal ions will excite the $d$ - $d$ transition in the internal $d$-orbit. This will allow the ligand field absorption band to be observed, and the energy required to produce this phenomenon is relatively low, so there was an absorption edge in the visible or near infrared region. Therefore, the figure shows characteristic bands at 600 to $650 \mathrm{~nm}$, which are typical for Co(II) ions [49]. The presence of Co(II) could be demonstrated by XPS. In addition, the absorption edge of ZIF-9/CuO 40\% showed an obvious red shift phenomenon compared with those of pure ZIF-9 and the blank group. Therefore, the addition of a $\mathrm{CuO}$ structure can enhance the light absorption intensity of the system and greatly improve the photocatalytic hydrogen production activity. Furthermore, the results of UV-vis spectroscopy could indirectly prove that the ZIF-9/CuO catalytic system was present.

\subsection{Mechanism of electron transfer}

To study the mechanism of electron transfer of the catalyst, the steady-state fluorescence spectra, obtained using an excitation wavelength of $468 \mathrm{~nm}$, of pure EY and EY-ZIF-9/CuO systems were analyzed. As shown in Fig. 11, the strongest emission peak of the system appeared at $537 \mathrm{~nm}$, with an intensity of $6.1 \times 10^{4}$ (a.u.). This prominent phenomenon indicated a quenching process of an EY electron of the excited state and strong recombination rate of electron-hole pairs [50]. When EY molecules adsorb energy, the electrons in the ground state will transition to the excited state. However, the lifetime of the electron in the excited state is very short and it returns to the ground state easily accompanied by radiative decay with the emission of photons, which results in the generation of fluorescence [51]. Therefore, it can be known that the stronger the fluorescence intensity, the worse the usage rate of the electron. Moreover, it can be seen from the figure that the decrease of the fluorescence intensity was in the order of EY, EY-ZIF-9 and the EY-ZIF-9/CuO (40\%) system. Moreover, there was no obvious overlap between the fluorescence emission peak of EY and the absorption peak of ZIF-9, so that the excited electrons of the EY could not be transferred from EY molecules to ZIF-9

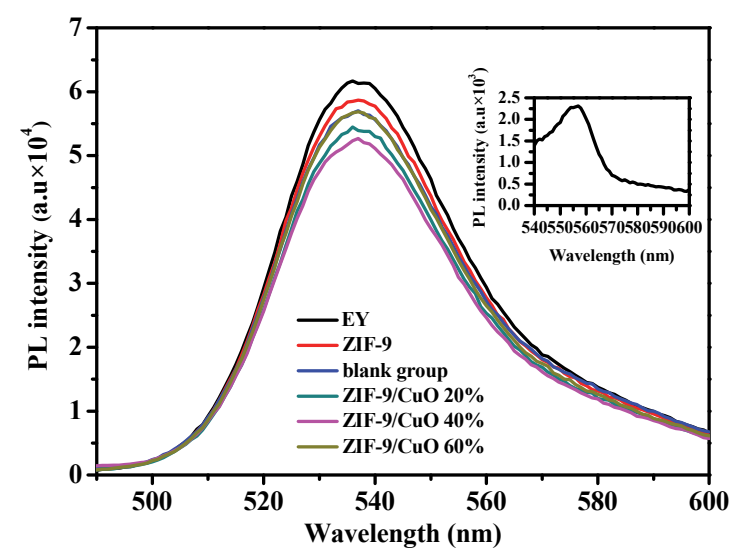

Fig. 11. Steady-state fluorescence spectra of EY and the EY-ZIF-9/CuO system in TEOA solution $(15 \% v / v)$ at $\mathrm{pH}=10$. in this reaction system [52]. Some of these electrons will directly reach the surface of the ZIF-9 surface, but more electrons will eventually reach the catalyst surface through $\mathrm{CuO}$, which will form hydrogen with the $\mathrm{H}^{+}$in the reaction solution. From the above explanation, we can conclude that the photocatalyst ZIF-9/CuO is present, and the addition of metal oxide $\mathrm{CuO}$ can improve the catalytic activity of the photocatalyst to some extent.

The fluorescence lifetimes of the EY, EY-ZIF-9, EY-blank group and EY-ZIF-9/CuO (40\%) were obtained by fitting the decay profiles to either a single-exponential or double-exponential. The relevant information is shown in Table 2. The fluorescence quenching efficiency of various materials was different. The quenching rate of EY-ZIF-9/CuO (40\%) was the largest, which reached $14 \%$. The lifetime of the dye molecule EY remained almost unchanged, which indicated that the fluorescence quenching of EY could be attributed to the static quenching mechanism. Hence, the excited dye was adsorbed onto the ZIF-9 surface and formed an association to induce the fluorescence quenching [53]. A difference was that the fluorescence lifetime of EY-ZIF-9/CuO (40\%) was drastically changed, and it indicated that the fluorescence decay of EY-ZIF-9/CuO (40\%) could be described by double-exponential fluorescence decay kinetics, namely a dynamic quenching mechanism. This phenomenon indicates that the addition of the metal oxide $\mathrm{CuO}$ in the reaction solution can enhance the aggregation of EY molecules. In comparison, the interaction between EY-ZIF-9/ $\mathrm{CuO}(40 \%)$ and $\mathrm{EY}$ molecules was greater than that of the EY-blank group and EY and that of EY-ZIF-9 and EY. Moreover, the strong interaction force was favorable for the adsorption of dyes and the transmission of photogenerated electrons. Thus, the photocatalytic hydrogen production efficiency was enhanced. In summary, the addition of copper oxide can make the catalyst more effective in accepting the transmission of photogenerated electrons and promoting the separation of photogenerated electron pairs, which thus enhances the photocatalytic hydrogen production performance.

The electron migration mechanism of the ZIF-9/CuO system was determined by the above fluorescence spectrum test. To further confirm the mechanism, the photoelectrochemical

Table 2

Decay parameters of EY in the presence of EY, EY-ZIF-9, EY-blank group and EY-ZIF-9/CuO (40\%) in 15\% $(v / v)$ TEOA aqueous solution at $\mathrm{pH}$ 10.

\begin{tabular}{lcccc}
\hline System ${ }^{\text {a }}$ & $\begin{array}{c}\text { Quchencing } \\
\text { efficiency } \\
(\%)\end{array}$ & $\begin{array}{c}\text { Lifetime } \\
(\tau, \mathrm{ns})\end{array}$ & $\begin{array}{c}\text { Average } \\
\text { lifetime } \\
(\tau, \mathrm{ns})\end{array}$ & $\chi^{2}$ \\
\hline EY b & - & 0.267 & 0.267 & 1.17 \\
EY-ZIF-9 b $^{\text {bY-blank group }}{ }^{\mathrm{b}}$ & 4.3 & 0.269 & 0.269 & 1.02 \\
EY-ZIF-9/CuO (40\%) c $^{c}$ & 7.0 & 0.272 & 0.272 & 1.05 \\
& 14.1 & $\tau_{1}=0.069$ & 0.298 & 1.04 \\
\hline
\end{tabular}

a Decay of a TEOA aqueous solution $(15 \% \mathrm{v} / \mathrm{v})$ of $1.0 \times 10^{-6} \mathrm{~mol} \mathrm{~L}^{-1} \mathrm{EY}$ at $\mathrm{pH} 10$ was recorded in the presence of $0.15 \mathrm{mg} \mathrm{mL}^{-1}$ EY, EY-ZIF-9, EY-blank group and EY-ZIF-9/CuO (40\%). The excitation and emission wavelengths were 468 and $537 \mathrm{~nm}$, respectively.

bSingle-exponential fit for EY, EY-ZIF-9, and EY-blank group.

c Double-exponential fit for EY-ZIF-9/CuO (40\%). 

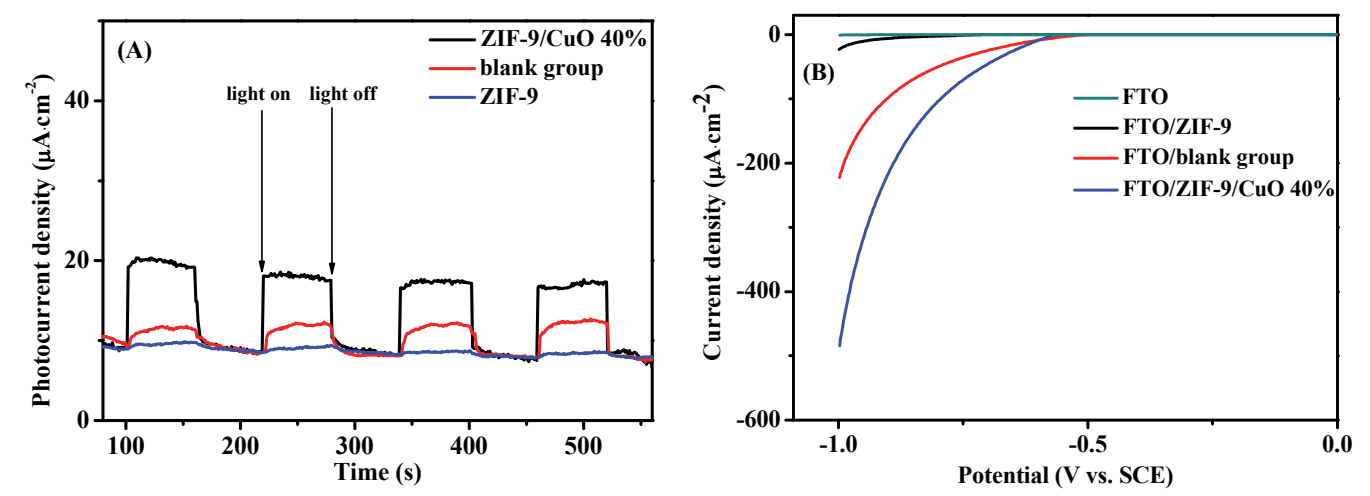

Fig. 12. (A) Transient photocurrent response for the EY-sensitized ZIF-9/CuO 40\%, blank group and ZIF-9 samples. (B) LSV curves of bare FTO glass and EY-sensitized ZIF-9/CuO 40\%, blank group and ZIF-9 coated in a mixed solution of $15 \mathrm{vol}_{2} \mathrm{TEOA}$ and $\mathrm{Na}_{2} \mathrm{SO}_{4}(0.1 \mathrm{~mol} \mathrm{~L}-1)$ at $\mathrm{pH} 10$. The scan rate was $1 \mathrm{mV} \mathrm{s}^{-1}$.

properties of the dye sensitized system in a mixed solution were determined. As shown in Fig. 12(A), the dye sensitized ZIF-9/CuO 40\%, blank group and ZIF-9 samples were uniformly coated on FTO and investigated under several on-off cycles of intermittent irradiation. The photocurrent response ability of ZIF-9/CuO 40\% was obviously superior under visible light while the response ability of the blank group was second, and the photocurrent response ability of ZIF-9 was last. This result was consistent with the above results. Moreover, this result further explained that the presence of $\mathrm{CuO}$ could effectively promote electron transfer from the strong reductive radical EY-• to ZIF-9/CuO and then to FTO. Thus, CuO could directly and effectively promote the separation of the photogenerated charge and improve the efficiency of the photocatalytic decomposition of hydrogen. Additionally, the photocurrent response ability of the blank group was slightly stronger than that of pure ZIF-9, which could be attributed to the action of cobalt oxide that resulted from the oxidation of methanol solution during deposition. Therefore, the addition of copper oxide is the key factor to change the catalytic system, and the photocatalytic reaction performance is improved by enhancing the photoelectric response ability.

In addition, the electrochemical $\mathrm{H}_{2}$ evolution activities of the ZIF-9/CuO 40\%, blank group and ZIF-9 coated on FTO glass were also investigated by using the linear sweep voltammetry (LSV) technique [54]. As shown in Fig. 12(B), the cathode current corresponding to the hydrogen ion could be seen on the FTO conductive glass, but the current density was still low at a higher voltage and the initial reduction potential was $-0.19 \mathrm{~V}$. By comparison, the reduction of the current density showed a gradual increase in the same voltage range on the FTO/ZIF-9, FTO/blank group and FTO/ZIF-9/CuO 40\% electrodes. This phenomenon showed that ZIF-9, blank group and ZIF-9/CuO $40 \%$ could all be used as a catalytic material for the electro-catalytic decomposition water to hydrogen. Further analysis showed that the starting reduction potential of the FTO/blank group and FTO/ZIF-9/CuO 40\% electrodes were almost the same at approximately $-0.53 \mathrm{~V}$. However, the most obvious difference was that the current density of the FTO/ZIF-9/CuO 40\% electrode was much larger than that of the FTO/blank group electrode at the same applied voltage of
-0.5 to $-0.1 \mathrm{~V}$, which indicated that the presence of $\mathrm{CuO}$ could further promote the electro-catalytic decomposition water to produce hydrogen with the blank group. This result was in line with the above conclusions.

According to the above experiment, characterization and conclusions, the reaction mechanism of $\mathrm{H}_{2}$ production over Eosin Y-sensitized ZIF-9/CuO photocatalysts under visible light irradiation is illustrated in Fig. 13. The photocatalytic activity of the ZIF-9/CuO photocatalyst could be increased by the addition of copper oxide, because ZIF-9 can provide a sufficient number of active sites for $\mathrm{CuO}$. Furthermore, more dye molecules could be adsorbed on the surface of the ZIF-9 to form singlet excited

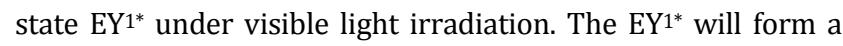
more stable triplet excited state $\mathrm{EY}^{3^{*}}$ through an intersystem transition. Then, the $\mathrm{EY}^{3^{*}}$ will be quenched to form the reduced state dye species $\mathrm{EY}^{-\bullet}$ arising from a strong reduction reaction, and the TEOA will become TEOA+[55]. Finally, the electrons of EY- $\bullet$ will be delivered to the catalyst that is connected to dye molecules. The vast majority of electrons will pass through ZIF-9 to $\mathrm{CuO}$ and combine with $\mathrm{H}^{+}$in the solution, owing to its electron transport characteristics. Meanwhile, the EY-• returns to the ground state, and the water reduction reaction is complete. In addition, a small amount of $\mathrm{H}^{+}$can also be reduced by EY-sensitized pure ZIF-9 to produce $\mathrm{H}_{2}$. Therefore, the excellent dye adsorption of ZIF-9 and excellent electronic acceptability of $\mathrm{CuO}$ can greatly enhance the photocatalytic activity,

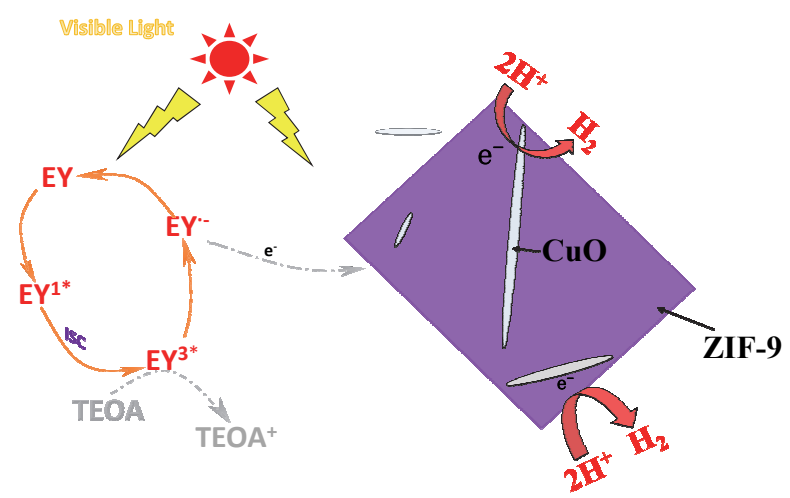

Fig. 13. Photocatalytic hydrogen production mechanism over EY sensitized ZIF-9/CuO under visible light irradiation. 
through the inhibition of the separation of the photogenerated charge, and accelerate the electron transfer rate.

\section{Conclusions}

A novel organic framework and metal-bound photocatalyst was successfully prepared at room temperature in a gentle manner. The $\mathrm{CuO}$ was successfully modified on the ZIF-9 by light irradiation deposition, which was further confirmed by EDX and XPS. The ZIF-9/CuO photocatalyst was effective for $\mathrm{H}_{2}$ generation under visible light with sacrificial agent conditions. When the $\mathrm{CuO}$ content was $40 \%$, the photocatalytic activity of the photocatalytic system was greatly improved, where the amount of hydrogen production could reach $78.74 \mu \mathrm{mol}$ under light irradiation for $5 \mathrm{~h}$. This arose from a sufficient number of active sites. By testing the stability of the photocatalyst, the addition of EY dyes was shown to be one of the main factors that affected the performance for continuous hydrogen production. The phenomenon further demonstrated the importance of light absorption. Fluorescence analysis provided a further understanding of the strong interaction between EY and ZIF-9/CuO (40\%), which was a dynamic quenching mechanism. Hence, the aggregation of the EY molecule on the catalyst surface was enhanced, so the catalytic activity of the photocatalyst could be increased. Moreover, a possible reaction mechanism was also proposed through photoelectrochemical characterization and the above analysis. The open-framework structure of ZIF-9 can adsorb more dye molecules and the $\mathrm{CuO}$, which connects with ZIF-9, can greatly improve the electronic transmission efficiency. From the above results, the combination of non-metallic organometallic frames and metal oxides for photocatalytic hydrogen production is shown to be feasible and promising.

\section{References}

[1] M. I. Hoffert, K. Caldeira, A. K. Jain, E. F. Haites, L. D. Harvey, S. D. Potter, M. E. Schlesinger, S. H. Schneider, R. G. Watts, T. M. L. Wigley, D. J. Wuebbles, Nature, 1998, 395, 881-884.

[2] D. D. Liu, Z. L. Jin, H. X. Li, G. X. Lu, Appl. Surf. Sci., 2017, 423, 255-265.

[3] Y. J. Cui, G. G. Zhang, Z. Z. Lin, X. C. Wang, Appl. Catal. B, 2016, 181, 413-419.

[4] A. Fujishima, K. Honda, Nature, 1972, 238, 37-38.

[5] J. P. Shi, R. Tong, X. B. Zhou, Y. Gong, Z. P. Zhang, Q. Q. Ji, Y. Zhang, Q. Y. Fang, L. Gu, X. N. Wang, Z. Liu, Y. F. Zhang, Adv. Mater., 2016, 28, 10664-10672.

[6] Q. Huang, J. G. Yu, S. W. Cao, C. Cui, B. Cheng, Appl. Surf. Sci., 2015, $358,350-355$.

[7] J. X. Low, J. G. Yu, M. Jaroniec, S. Wageh, A. A. Al-Ghamdi, Adv. Mater., 2017, 29, 1601694.

[8] M. S. Akple, J. X. Low, Z. Qin, S. Wageh, A. A. Al-Ghamdi, J. G. Yu, S. W. Liu, Chin. J. Catal., 2015, 36, 2127-2134.

[9] M. Dinc, A. F. Yu, J. R. Long, J. Am. Chem. Soc., 2006, 128, 8904-8913.

[10] O. M. Yaghi, M. O. Keeffe, N. W. Ockwig, H. K. Chae, M. Eddaoudi, J. Kim, Nature, 2003, 423, 705-714.

[11] L. J. Murray, M. Dinc, J. R. Long, Chem. Soc. Rev., 2009, 38, 1294-1314.
[12] H. C. Zhou, J. R. Long, O. M. Yaghi, Chem. Rev., 2012, 112, 673-674.

[13] M. Alvaro, E. Carbonell, B. Ferrer, F. X. Llabrés i. Xamena, H. Garcia, Chem. Eur. J., 2007, 13, 5106-5112.

[14] N. W. Ockwig, O. Delgado-Friedrichs, M. O’Keefe, O. M. Yaghi, Acc. Chem. Res., 2005, 38, 176-182.

[15] C. Wang, Z. G. Xie, K. E. Clekraff, W. B. Lin, J. Am. Chem. Soc., 2011, 133, 13445-13454.

[16] Y. H. Fu, D. R. Sun, Y. J. Chen, R. K. Huang, Z. X. Ding, X. Z. Fu, Z. H. Li, Angew. Chem. Int. Ed., 2012, 51, 3364-3367.

[17] L. J. Shen, S. J. Liang, W. M. Wu, R. W. Liang, L. Wu, J. Mater. Chem. A, 2013, 1, 11473-11482.

[18] Y. P. Yuan, L. S. Yin, S. W. Cao, G. S. Xu, C. H. Li, C. Xue, Appl. Catal. B, 2015, 168, 572-576.

[19] L. He, L. Li, T. T. Wang, H. Gao, G. Z. Li, X. T. Wu, Z. W. Su, C. G. Wang, Dalton Trans., 2014, 43, 16981-16985.

[20] S. Hashemian, A. Sedrpoushan, F. H. Eshbala, Catal. Lett., 2017, 147, 196-203.

[21] S. Hermes, M. K. Schröter, R. Schmid, L. Khodeir, M. Muhler, A. Tissler, R. A. Fischer, Angew. Chem. Int. Ed., 2005, 44, 6237-6241.

[22] J. M. Yan, Z. L. Wang, L. Gu, S. J. Li, H. L. Wang, W. T. Zheng, Q. Jiang, Adv. Energy Mater., 2015, 5, 1500107/1-1500107/6.

[23] W. T. Koo, S. J. Choi, S. J. Kim, J. S. Jang, H. L. Tuller, I. D. Kim, J. Am. Chem. Soc., 2016, 138, 13431-13437.

[24] L. J. Shen, R. W. Liang, L. Wu, Chin. J. Catal., 2015, 36, 2071-2088.

[25] W. Chaikittisilp, N. L. Torad, C. Li, M. Imura, N. Suzuki, S. Ishihara, Y. Yamauchi, Chem. Eur. J., 2014, 20, 4217-4221.

[26] Q. M. Li, H. Kim, Fuel Process Technol., 2012, 100, 43-48.

[27] Q. Q. Hu, J. Q. Huang, G. J. Li, Y. B. Jiang, H. Lan, W. Guo, Y. G. Cao, Appl. Surf. Sci., 2016, 382, 170-177.

[28] M. Dong, P. Zhou, C. J. Jiang, B. Cheng, J. G. Yu, Chem. Phys. Lett., 2017, 668, 1-6.

[29] Y. F. Li, W. P. Zhang, X. Shen, P. F. Peng, L. B. Xiong, Y. Yu, Chin. J. Catal., 2015, 36, 2229-2236.

[30] H. W. Wu, S. Y. Lee, W. C. Lu, K. S. Chang, Appl. Surf. Sci., 2015, 344, 236-241.

[31] J. W. Fu, S. W. Cao, J. G. Yu, J. Materiomics, 2015, 1, 124-133.

[32] G. H. Li, N. M. Dimitrijevic, L. Chen, T. Rajh, K. A. Gray, J. Phys. Chem. C, 2008, 112, 19040-19044.

[33] Z. J. Li, Y. Qu, G. He, M. Humayun, S. Y. Chen, L. Q. Jing, App. Surf. Sci., 2015, 351, 681-685.

[34] H. J. Choi, M. Kang, Int. J. Hydrogen Energy, 2007, 32, 3841-3848.

[35] R. Michal, E. Dworniczek, M. Caplovicova, O. Monfort, P. Lianos, L. Caplovic, G. Plesch, Appl. Surf. Sci., 2016, 371, 538-546.

[36] Q. Q. Hu, J. Q. Huang, G. J. Li, J. Chen, Z. J. Zhang, Z. H. Deng, Y. B. Jiang, W. Guo, Y. G. Cao, Appl. Surf. Sci., 2016, 369, 201-206.

[37] Y. Q. Wang, T. T. Jiang, D. W. Meng, D. G. Wang, M. H. Yu, Appl. Surf. Sci., 2015, 355, 191-196.

[38] S. P. Xu, D. D. Sun, Int. J. Hydrogen Energy, 2009, 34, 6096-6104.

[39] Z. K. He, J. W. Fu, B. Cheng, J. G. Yu, S. W. Cao, Appl. Catal. B, 2017, 205, 104-111.

[40] S. R. Venna, J. B. Jasinski, M. A. Carreon, J. Am. Chem. Soc., 2010, 132, 18030-18033.

[41] L. T. L. Nguyen, K. K. A. Le, H. X. Truong, N. T. Sphan, Catal. Sci. Technol., 2012, 2, 521-528.

[42] B. X. Li, Y. F. Wang, Superlattice Microstruct., 2010, 47, 615-623.

[43] X. Q. Du, J. W. Huang, Y. Y. Feng, Y. Ding, Chin. J. Catal., 2016, 37, 123-134.

[44] Y. Y. Cui, Y. X. Wang, H. Wang, F. Cao, F. Y. Chen, Chin. J. Catal., 2016, 37, 1899-1906.

[45] K. Kalyanasundaram, J. Kiwi, M. Grätze, Helv. Chim. Acta, 1978, 61, 2720-2730.

[46] S. X. Min, G. X. Lu, Int. J. Hydrogen Energy, 2012, 37, 10564-10574. 


\title{
Graphical Abstract
}

Chin. J. Catal., 2017, 38: 2056-2066 doi: 10.1016/S1872-2067(17)62969-3

Construction of CuO-modified zeolitic imidazolate framework-9 for photocatalytic hydrogen evolution

Kai Fan, Zhiliang Jin*, Hong Yuan, Hongyan Hu*, Yingpu Bi North Minzu University;

Lanzhou Institute of Chemical Physics, Chinese Academy of Science

A photocatalyst containing cube ZIF- 9 and $\mathrm{CuO}$ is synthesized and shows a high photocatalytic activity that is 19.4 times that of $\mathrm{g}-\mathrm{C}_{3} \mathrm{~N}_{4} / \mathrm{CuO}$. The large specific surface area of ZIF-9 plays an important role.

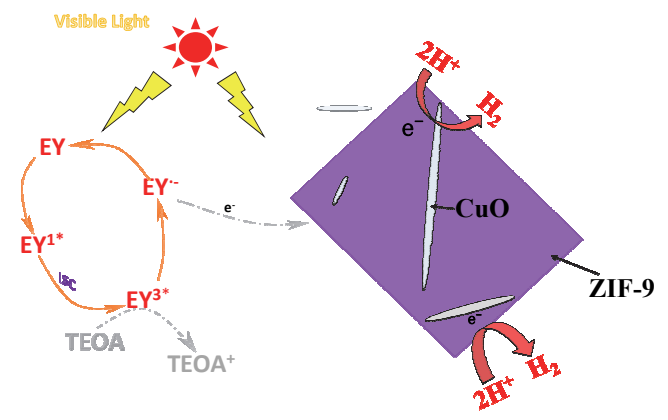

[47] S. G. Kumar, K. S. R. K. Rao, Appl. Surf. Sci., 2017, 391, 124-148.

[48] X. Q. Hao, Z. L. Jin, S. X. Min, G. X. Lu, $R S C A d v ., 2016,6$, 23709-23717.

[49] M. Tonigold, Y. Lu, B. Bredenkötter, B. Rieger, S. Bahnmüller, J. Hitzbleck, G. Langstein, D. Volkmer, Angew. Chem. Int. Ed., 2009, 48, 7546-7550.

[50] Q. Y. Li, L. Chen, G. X. Lu, J. Phys. Chem. C, 2007, 111, 11494-11499.
[51] Q. Y. Li, Z. L. Jin, Z. G. Peng Y. X. Li, S. B. Li, G. X. Lu, J. Phys. Chem. C, 2007, 111, 8237-8241.

[52] Y. Liu, C. Y. Liu, Y. Liu, Appl. Surf. Sci., 2011, 257, 5513-5518.

[53] S. X. Min, G. X. Lu, J. Phys. Chem. C, 2012, 116, 25415-25424.

[54] Y. Liu, C. Liu, Y. Liu, Appl. Surf. Sci., 2011, 257, 5513

[55] X. Q. Hao, Z. L. Jin, F. Wang, J. Xu, S. X. Min, H. Yan, G. X. Lu, Superlattice Microstruct., 2015, 82, 599-611.

\section{$\mathrm{CuO}$ 修饰改性沸石咪唑框架催化剂的构建及其光催化产氢性能}

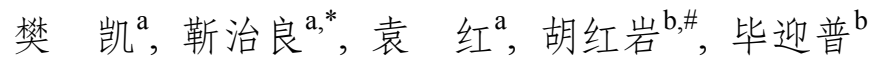 \\ a 北方民族大学化学与化学工程学院, 宁夏银川 750021

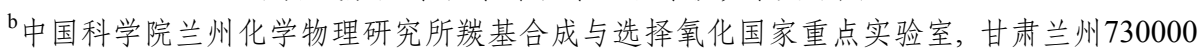

\begin{abstract}
摘要: 新型金属有机骨架 $(\mathrm{MOFs})$ 材料是一类比较新颖的可用作光催化剂的潜在材料. 它是由金属离子或金属簇单元与有 机配体通过配位作用自组装形成的一类具有周期性多维网络结构的多孔晶态材料. MOFs结构具有高度的有序性、一定程 度上的可设计性和可剪裁性, 其高度发达的孔结构使得它具有超高的比表面积. MOFs结构中的金属簇被认为可以扮演半 导体量子点的角色, 同时其有机配体基于“天线效应”, 在光激发条件下用来活化这些金属簇, 从而使得MOFs成为可能的光 催化剂.

ZIF-9 由角共享四面体 $\mathrm{CoN}_{4}$ 单元组成, 其中 $\mathrm{Co}^{2+}$ 阳离子和苯并咪唑阴离子之间的配位键是 $\mathrm{N}$ 供体配体, 因而具有良好的 热稳定性和较高的碳氮含量. 本文在一定浓度的甲醇溶液中通过光沉积法将 CuO附着于ZIF-9表面上, 用于光催化活性测 试, 并与 $g-\mathrm{C}_{3} \mathrm{~N}_{4} / \mathrm{CuO}$ 催化剂进行了比较. 结果表明, ZIF-9/CuO催化剂活性是 $\mathrm{g}-\mathrm{C}_{3} \mathrm{~N}_{4} / \mathrm{CuO}$ 催化剂的 19.4 倍. 这是由于比表面 积的不同, 导致所吸附的染料与暴露的活性位点的不同. 通过SEM, TEM, EDS, XRD, XPS, UV-Vis和稳态瞬态荧光等手段 系统研究了催化剂反应动力学行为及内在机理. 稳态荧光结果表明, 染料的荧光发射峰和ZIF-9的吸收峰之间没有明显的 重叠, 染料的激发电子可以在该反应系统中从染料分子转移到ZIF-9上. 除了一部分电子直接传输到达ZIF-9表面, 更多的 电子将最终通过 $\mathrm{CuO}$ 到达催化剂表面, 最后与反应溶液中的 $\mathrm{H}^{+}$结合生成 $\mathrm{H}_{2}, \mathrm{EY}-\mathrm{ZIF}-9 / \mathrm{CuO}(40 \%)$ 的荧光衰减可以用双指数 荧光衰减动力学即动态淬火机理. 表明 $\mathrm{CuO}$ 的引入可以增强染料 $\mathrm{EY}$ 分子的聚集, 从而增加该催化剂活性.
\end{abstract}

关键词: ZIF-9; $\mathrm{CuO}$; 染料; 敏化途径; 产氢; 反应优化

收稿日期: 2017-10-01. 接受日期: 2017-10-31. 出版日期: 2017-12-05.

*通讯联系人. 电话: 13893316102; 传真: (0951)2067915; 电子信箱: zl-jin@nun.edu.cn

\#通讯联系人. 电子信箱: HuHongyan@licp.cas.cn

基金来源：国家自然科学基金 $(21433007,21603247,41663012)$; 北方民族大学 “宁夏低品位资源高值化利用及环境化工一体化技 术”创新团队项目.

本文的电子版全文由Elsevier出版社在ScienceDirect上出版(http://www.sciencedirect.com/science/journal/18722067). 